\title{
Infectious keratitis in patients undergoing Boston Type 1 keratoprosthesis (Boston KPro) procedure: case series
}

\author{
Ceratite infecciosa em pacientes submetidos ao implante de ceratoprótese \\ Boston Tipo I: série de casos
}

Heloisa Moraes do Nascimento ${ }^{1}$, Lauro Augusto de Oliveira ${ }^{2}$, Ana Luisa Höfling-Lima ${ }^{1}$

\begin{abstract}
Description of two cases of infectious keratitis in patients after Boston Type 1 keratoprosthesis (Boston KPro) implantation. The first case refers to a patient that had the device indicated due to limbal deficiency secondary to severe dry eye who presented a fungal infection by Aerobasidium pullulans that was successfully treated with amphotericin B eye drops. The second case reports a patient with Boston KPro implantation due to previous corneal transplant rejection showing bacterial keratitis in the fourth postoperative month. The etiologic agent was identified as Streptococcus $s p$ and topical treatment with vancomycin was effective. The importance of postoperative surveillance in Boston KPro eyes is discussed.
\end{abstract}

Keywords: Cornea/surgery; Corneal diseases/surgery; Keratitis; Prosthesis implantation; Visual acuity; Preoperative care; Human; Female; Aged, 80 and over; Case reports

\section{RESUMO}

Descrição de dois casos de ceratite infecciosa em pacientes submetidos a implante de ceratoprótese Boston Tipo 1 (Boston KPro). O primeiro caso refere-se a uma pacientena qual o dispositivo foi indicado por deficiência límbica secundária a olho seco grave, no qual foi identificado infecção fúngica por aerobasidium pullulans, tratada com sucesso com colírio de anfotericina B. O segundo caso reporta uma paciente com implante de Boston KPro por falências de transplantes de córnea prévios, que apresentou ceratite bacteriana no quarto mês pós-operatório. O agente etiológico identificado foi Streptococcus sp e o tratamento tópico com vancomicina foi eficaz. Discute-se a importância da vigilância pós-operatória em olhos submetidos ao implante de ceratoprótese.

Descritores: Córnea/cirurgia; Doenças da córnea/cirurgia;Ceratite; Implante deprótese; Acuidade visual; Cuidados pré operatórios; Humanos; Feminino; Idoso de 80 anos ou mais; Relatos de casos

\section{INTRODUCTION}

Keratoprosthesis (KPro) surgery is usually indicated for patients with multiple corneal transplant rejection and also for patients with cicatricial ocular surface disease (autoimmune diseases, chemical and thermal eye burns, etc) ${ }^{(1)}$. Considering the severity of ocular surface involvement in these patients, as well as the numerous surgical procedures that they have undergone, the high risk of postoperative complications in these patients is understood. These complications include: glaucoma ${ }^{(1)}$, tissue melting leaking aqueous humor ${ }^{(2)}$, device extrusion, chronic inflammation and retroprothetic membrane formation ${ }^{(3)}$, retinal detachment ${ }^{(4)}$, macular edema, infectious keratitis and bacterial endophthalmitis ${ }^{(5-6)}$.

Boston type $1 \mathrm{KPro}$ is used in patients with relatively normal eyelid function and ability to maintain the ocular surface "wet". After Boston KPro implantation, the corneal surface becomes vulnerable to evaporative forces. Epithelial defects, stromal thinning, dellen formation and melting, especially in eyes with underlying inflammatory disease may occur ${ }^{(7)}$.

Since the beginning of Boston KPro use, one of the problems related to the device was dehydration of ocular surface. This issue was successfully solved with the use of therapeutic contact lenses in the eyes implanted with Boston Type 1 KPro. However, the use of contact lens increases the risk of corneal infection and colonization and, therefore, it requires additional care of physician and patient. The use of therapeutic contact lens improved quality of the ocular surface in Boston KPro users and was also responsible for increased indications of this procedure worldwide.

The increased risk of ocular infection in patients with tear film dysfunction and in patients receiving chronic topical corticosteroids justified the need to establish a prophylactic antibiotic regimen for patients with Boston Type 1 KPro implant. Previously, the prophylactic regimen consisted of polymyxin-trimethoprim, gentamicin or ofloxacin. However, the large number of infections even using this prophylactic regimen caused its modification to the use of broad-spectrum antibiotics. Currently, vancomycin is used in the United States as a prophylactic antibiotic regimen of choice for Boston KPro patients ${ }^{(8)}$.

The prophylactic use of broad-spectrum antibiotics associated with contact lens predisposes to fungal colonization and infection in Boston KPro eyes ${ }^{(9)}$. Warm and humid environments are other predisposing factors for fungal infection and colonization ${ }^{(9)}$. This weather pattern is very common in Brazil. 
Although recommended by the manufacturer, prophylaxis with vancomycin eyedrops has some limitations in our country, such as cost of handling and storage difficulties. The Cornea and External Eye Disease Sector, Department of Ophthalmology of UNIFESP uses $0.5 \%$ moxifloxacin as prophylaxis associated with programmed replacement of in patients with Boston Type 1 keratoprosthesis implantation.

\section{CASE REPORT}

\section{CASE 1}

TAC, a 94-year old female diagnosed with limbic failure secondary to severe dry eye, underwent Boston Type 1 keratoprosthesis implantation in the right eye.

According to postoperative routine of the service, the patient was assessed on the first postoperative follow-up, after two weeks and then monthly. The postoperative regimen included 0.5\% moxifloxacin as prophylaxis, topical steroids, lubricating eye drops and contact lenses (Kontur Contact Lens, Richmond, CA). This hydrophilic contact lens was suggested by the Boston KPro manufacturer and its replacement programmed to every three months. The steroids were reduced gradually according to ocular inflammation, but maintained one drop daily. The authors cannot assure about patients compliance regarding the use of antibiotic and the prophylactic regimen, but they were exhaustively informed about it. Cultures of the conjunctiva and of the discarded therapeutic contact lenses were collected for routine monitoring.
Two months after surgery, the patient had a positive culture for fungus Aerobasidium pullulans in the analysis of contact lenses. However, this analysis did not show any visible fungal colonization in the contact lens and the biomicroscopy showed no evidence suggesting infection.

As there were no obvious signs of eye infection and nor Boston Kpro or contact lens colonization, an expectant management was used. Two weeks after the positive culture found in the therapeutic contact lens, the patient developed a slight opacity extending from 3 to 5 hours at the interface between the anterior plate of keratoprosthesis and the donor corneal button (Figure 1).

At that time, due to the presence of visible corneal infiltration, antifungal eyedrops were initiated. Patient was treated with $0.15 \%$ amphotericin B eyedrops q.i.d.

The patient was subsequently monitored and showed regression of corneal opacity.

\section{CASE 2}

TMF, an 83-year old female diagnosed with two previous episodes of corneal transplant failure, underwent Boston Type 1 keratoprosthesis implantation in the right eye.

The same postoperative care previously described for the patient mentioned above was conducted in this patient.

Four months after surgery, the patient developed corneal opacity on the entire interface between the anterior plate of Boston KPro and donor corneal button. There was denuded epithelium in the
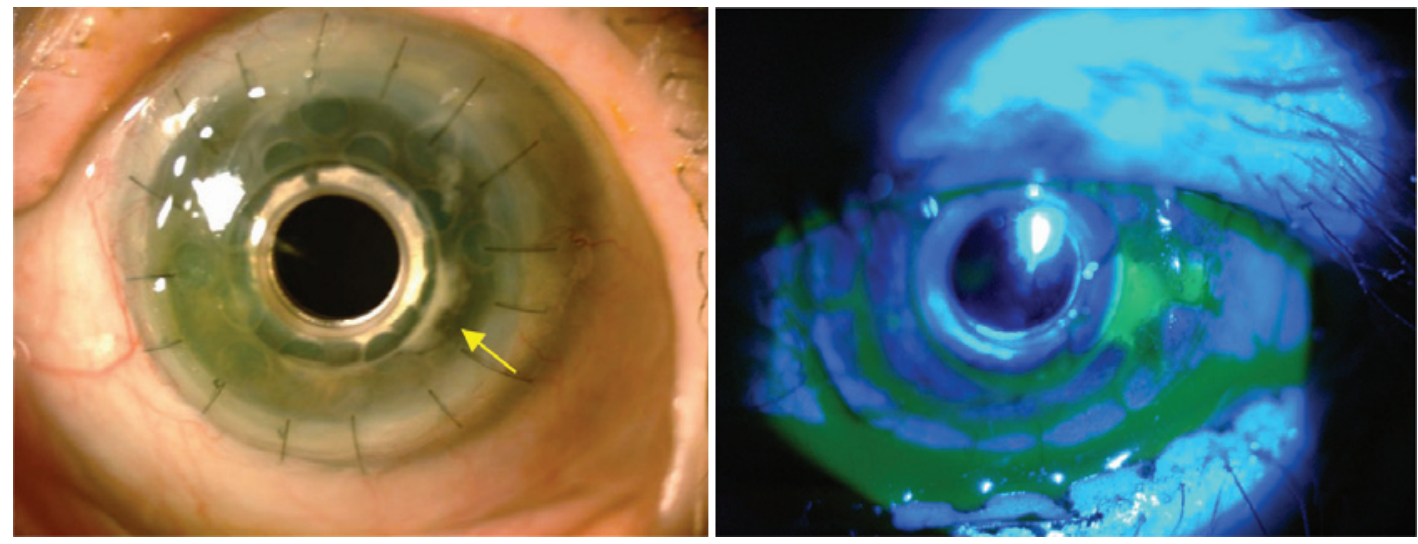

Figure 1. Opacity at the interface between anterior plate of the KPro and donor corneal button. Note the epithelial denuding area evidenced by fluorescein staining under cobalt blue light.
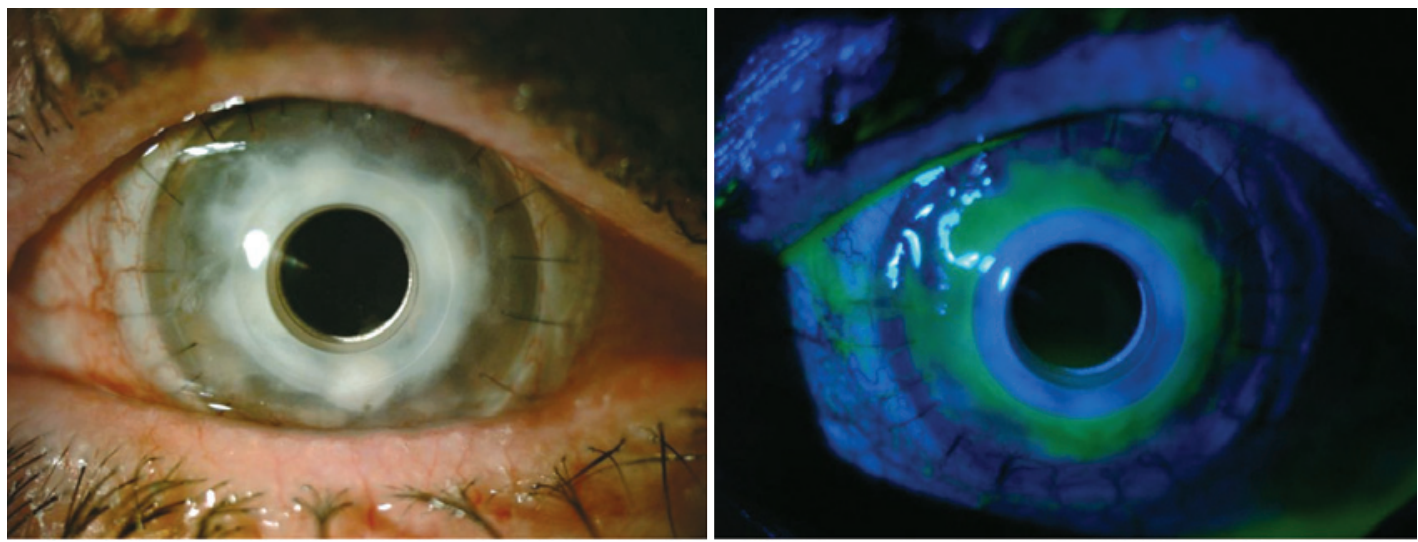

Figure 2. Corneal infiltrate in the full extension of the interface between anterior plate of KPro and donor corneal button. 
opaque area, as shown in figure 2. The culture of corneal scrapings from the affected area was positive for Streptococcus $s p$.

At that point, considering the continued use of $0.5 \%$ moxifloxacin concomitant with the corneal infiltrate onset and the fact that the microorganism was resistant to moxifloxacin, treatment with $5 \%$ vancomycin eye drops was initiated. There was gradual improvement after seven days of treatment, with disappearance of the corneal infiltrate and epithelial healing.

\section{DISCUSSION}

As far as we know, this is the first report of fungal isolation in the ocular surface of patients after Boston Type 1 keratoprosthesis implantation in Brazil. According to Barnes et al., the rate of fungal colonization in Boston KPro eyes is about $10 \%{ }^{(9)}$. This incidence was similar even before the use of broad-spectrum antibiotics and contact lenses ${ }^{(5)}$, and was also similar to those found in normal conjunctival flora, with a fungal colonization ranging from 5 to $25 \%{ }^{(9)}$. Fungal infections cannot be predicted by routine periodic cultures of the ocular surface of Boston KPro eyes as reported by Barnes et al that fungal colonization fluctuates spontaneously over the time ${ }^{(9)}$.

In the first case, fungal growth was found two months after Boston Type 1 keratoprosthesis implantation. This might have been favored by the tropical climate in Brazil, the prophylactic regimen (broad spectrum antibiotic and steroids) and the use of contact lenses.

Antifungal prophylactic regimen should not be recommended for all Boston keratoprosthesis users due to the low incidence of fungal infections and the lack of correlation between the routine surveillance cultures and the emergence of infections ${ }^{(9)}$. However, initiation of aggressive antifungal regimen is recommended in three circumstances: visible corneal colonization; visible colonization in the therapeutic contact lens and presence of active infection.

As the patient developed a visible corneal infiltration, the antifungal treatment was initiated, resulting in clinical improvement.

In the second case, a bacterial infection was diagnosed in the routine postoperative assessment. The rapid identification of the causative agent and the initiation of appropriate treatment were crucial to good clinical response and maintenance of keratoprosthesis.
The use of contact lens, trauma, ocular surface disease and corneal surgery are the major risk factors for infectious keratitis ${ }^{(10)}$.

Among the four main risk factors for the development of infectious keratitis, the second patient of the case series showed three of them. Importantly, the site most prone to developing infectious keratitis in patients after Boston Type 1 keratoprosthesis implantation is between anterior plate of prosthesis and the donor button, where there is a virtual space in which the tear film distribution is irregular, favoring the accumulation of tear and, consequently, the proliferation of microorganisms.

Keratoprosthesis is a promising device in modern corneal surgery. However, the high rate of risk factors for infections requires a thorough postoperative care, with surveillance cultures and routine monitoring visits. This case series is an example of the importance to keep Boston KPro patients on an exhaustive and close postoperative regimen in order to recognize the onset of infections. Any sign of infection should be promptly investigated and tailored treated to promote greater durability of the device.

\section{REFERENCES}

1. Netland PA, Terada H, Dohlman CH. Glaucoma associated with keratoprosthesis. Ophthalmology. 1998;105(4):751-7.

2. Yaghouti F, Nouri M, Abad JC, Power WJ, Doane MG, Dohlman CH.Keratoprosthesis: preoperative prognostic categories. Cornea. 2001:20(1):19-23.

3. Dohlman CH, Abad JC, Dudenhoefer EJ, Graney JM. Keratoprosthesis: beyond corneal graft failure. In: Spaeth GL, editor. Ophthalmic surgery: principles and practice. $3^{\text {rd }}$ ed. Philadelphia:WB Sanders; 2002. p.199-207.

4. Ray S, Khan BF, Dohlman CH, D'Amico DJ. Management of vitreoretinal complications in eyes with permanent keratoprosthesis. Arch Ophthalmol. 2002;120(5):559-66.

5. Nouri M, Terada H, Alfonso EC, Foster CS, Durand ML, Dohlman CH. Endophthalmitis after keratoprosthesis: Incidence, bacterial etiologies, and risk factors. Arch Ophthalmol. 2001; 119(4):484-9.

6. Dohlman CH, Barnes SD, Ma JK. Keratoprosthesis. In: Krachmer JH, Mannis MJ, Holland EJ, editors. Cornea. 2nd ed. Edinburgh: Elsevier; 2005. p.1719-28.

7. Dohlman CH, Dudenhoefer EJ, Khan BF, Morneault S. Protection of the ocular surface after keratoprosthesis surgery: the role of soft contact lenses. CLAO J. 2002;28(2):72-4.

8. Dohlman CH, Barnes SD, Nouri M. Preventing endophthalmitis in keratoprostheses: prophylactic antibiotic regimens. Invest Ophth Vis Sci. 2003;44:S108.

9. Barnes SD, Dohlman CH, Durand ML. Fungal colonization and infection in Boston keratoprosthesis. Cornea. 2007;26(1):9-15.

10. Khan BF, Harissi-Dagher M, Khan DM, Dohlman CH. Advances in Boston keratoprosthesis: enhancing retention and prevention of infection and inflammation. Int Ophthalmol Clin. 2007;47(2):61-71. Review.

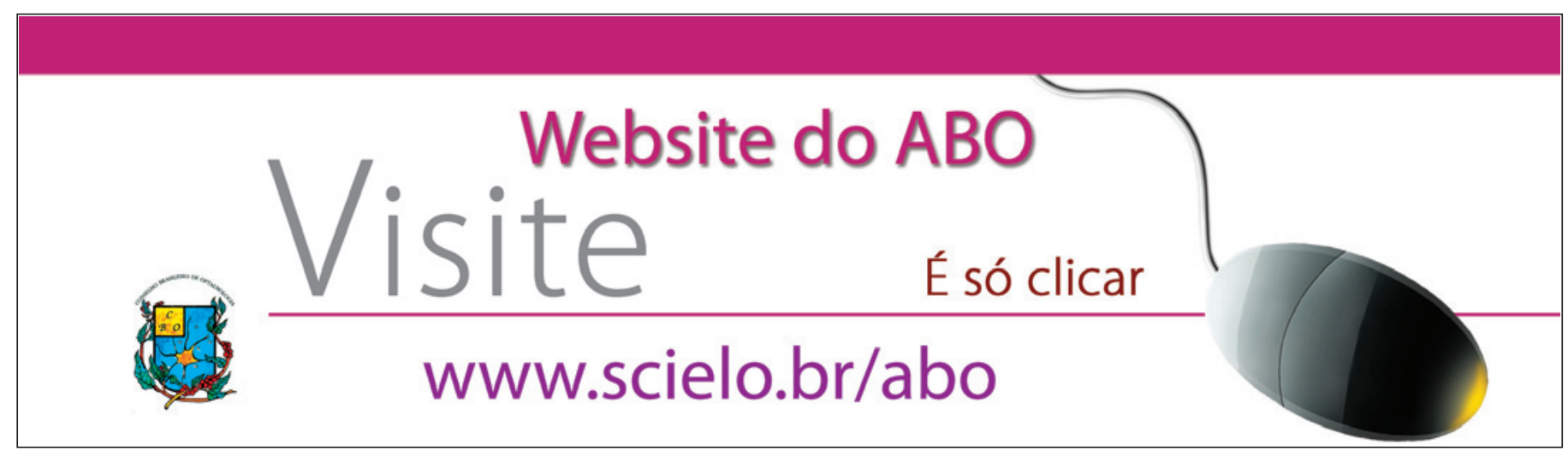

